\title{
A Semantic-Based Topic Knowledge Map System (STKMS) for Lesson-Learned Documents Reuse in Product Design
}

\author{
Ywen HUANG $^{\dagger \text { a) }}$, Student Member and Zhua JIANG ${ }^{\dagger}$, Nonmember
}

\begin{abstract}
SUMMARY In the process of production design, engineers usually find it is difficult to seek and reuse others' empirical knowledge which is in the forms of lesson-learned documents. This study proposed a novel approach, which uses a semantic-based topic knowledge map system (STKMS) to support timely and precisely lesson-learned documents finding and reusing. The architecture of STKMS is designed, which has five major functional modules: lesson-learned documents pre-processing, topic extraction, topic relation computation, topic weights computation, and topic knowledge map generation modules. Then STKMS implementation is briefly introduced. We have conducted two sets of experiments to evaluate quality of knowledge map and the performance of utilizing STKMS in outfitting design of a ship-building company. The first experiment shows that knowledge maps generated by STKMS are accepted by domain experts from the evaluation since precision and recall are high. The second experiment shows that STKMS-based group outperforms browse-based group in both learning score and satisfaction level, which are two measurements of performance of utilizing STKMS. The promising results confirm the feasibility of STKMS in helping engineers to find needed lesson-learned documents and reuse related knowledge easily and precisely.

key words: knowledge management, topic knowledge map, lesson-learned documents, semantic
\end{abstract}

\section{Introduction}

Product design is becoming increasingly knowledge intensive in manufacturing enterprises [1]. Many knowledge managing models and tools have been proposed and implemented to promote knowledge sharing among engineers in a collaborative team environment, which can improve efficiency of product design [2]. In the process of product design, engineers usually write lesson-learned documents for empirical knowledge sharing to others, and lots of empirical knowledge are in the forms of lesson-learned documents (see Fig. 3). When an engineer wants to learn from others' empirical knowledge, he or she will try to find and read others' lesson-learned documents which are textual materials, nevertheless, it may be not efficient for the engineer to find the proper files and reuse corresponding knowledge, especially in the following three situations. First, there are usually lots of lesson-learned documents which are stored in the hard disk of local network of an enterprise, but engineers can only search by the keywords of the file name, not the document content, and the results are inaccurate [3]. Second, when engineers want to look for hints or inspirations from lesson-learned documents, they have to read them from

Manuscript received June 24, 2013.

Manuscript revised October 16, 2013.

${ }^{\dagger}$ The authors are with the Dept. of Industrial Engineering, Shanghai Jiao Tong Univ., P.R. China.

a) E-mail: hywen2004@163.com (Corresponding author) DOI: 10.1587/transinf.E97.D.1049 beginning to end one by one, if under time pressure engineers can not find a specific way to distinguish needed documents. Third, it is difficult for engineers to recognize topics and relations among different lesson-learned documents, although these documents may be relevant on the subjects and themes.

In this paper, a novel approach, using a semanticbased topic knowledge map system (STKMS), supporting graphic-based topics and relations representation of lessonlearned documents, is proposed to assist engineers to reuse empirical knowledge. The core of STKMS is topic knowledge map. Key topics and relations of lesson-learned documents can be recognized directly from the map, so with the navigation of STKMS, knowledge demanders can identify their needed topics and find the proper lesson-learned documents with minimum effort.

The rest of paper is organized as follows. Some related works done by other scholars are briefly introduced in the next section. In Sect. 3, we propose the general architecture of STKMS, and the detailed procedure of the semanticbased topic knowledge map creation method is given. The implementation and scenario of STKMS for lesson-learned documents in outfitting design is introduced in Sect. 4. Two sets of experiments are conducted. The fisrt experiment in Sect. 5 is to validate the proposed knowledge map creation method. The other experiment in Sect. 6 is to evaluate the performance of utilizing STKMS. Closing remark and future work are then outlined in the last section.

\section{Related Works}

Topic knowledge map for knowledge representation and visualization is a hot topic in the scope of knowledge management. Several models and approaches on topic knowledge map have been studied since last decade [4]-[6]. Topic maps have been used in the e-learning domain as a content management technology. Stanescu et al present original ways of using topic maps for information structuring and retrieval in medical e-learning domain [7]. Topic map graphical view allows learner navigation for studying the topics and associations between them, and topic map querying using tolog that facilitates the establishing of search criteria for learning resources filtering [8]. Mihai et al propose an original algorithm for automated building of a topic map starting from a relational database of an e-learning system [9].

Topic map is rapidly becoming an industrial standard for knowledge representation with a focus for later search 
and extraction. The documents are transformed into a topic map based coded knowledge and the similarity between a pair of documents is represented as a correlation between the common patterns (sub-trees) [10]. A topic map model meets the need of organizing contents from various resources (documents, databases, videos, etc.) and from different languages. An incremental construction approach of multilingual topic maps is proposed, which main goal is to facilitate user's navigation across documents available in different languages [11]. Lu et al propose a method to provide a new view of text and help to find new knowledge and provide a multi-layer, visual knowledge map, available for users to acquire the knowledge and associations among them [12]. Newman et al explore visualizations of document collections by topic maps, where the topic model is used to determine the semantic content of each document. Using two collections of search results, they show how topic maps reveal the semantic structure of a collection and visually communicate the diversity of content in the collection [13].

Previous work mostly focused on automatic construction of knowledge maps based on statistics analysis technique, such as pure topic frequency analysis, etc. In the present study, the semantic relevancies among topics are considered. We propose a novel semantic-based method of topic knowledge map generation from lesson-learned documents and utilize STKMS to navigate lesson-learned documents for knowledge reusing. An implementation example with real-world data in outfitting design of a ship-building company is presented.

\section{Architecture of STKMS}

When performing product design activities, engineers can utilize STKMS to orientate the lesson-learned documents and get the related empirical knowledge, which could save a lot of time and energy. The system should have the abilities to generate a topic knowledge map automatically according to all the collected lesson-learned documents. The core of STKMS is semantic-based topic knowledge map. By using STKMS, engineers could have a whole picture, by which topics of all the lesson-learned documents and their relations among topics will show. Moreover, users could adjust the system input by adding, or changing the volume and sorts of lesson-learned documents, and the topic knowledge map will change. Besides the semantic-based topic knowledge map, our STKMS combines natural language processing and document clustering technologies, etc. This section details the STKMS's architecture and the creation method of semantic-based topic knowledge map.

The architecture of STKMS is depicted in Fig. 1, which has five major functional modules, including lesson-learned documents preprocessing, topic extraction, topic relation computation, topic weights computation, and topic knowledge map generation modules. The details of each module are described as follows.

(1) Lesson-learned documents preprocessing module: This module is responsible for determining morpholog-

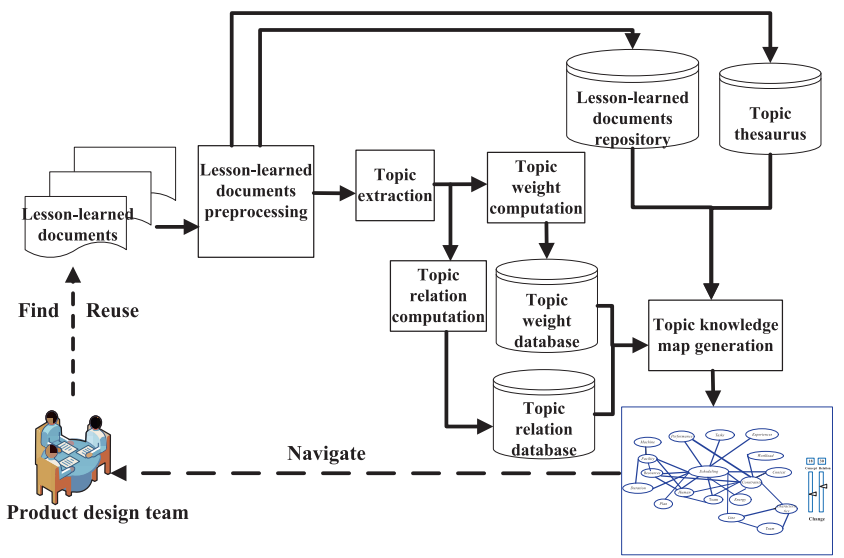

Fig. 1 STKMS architecture for the product design team.

ical features of lesson-learned documents of product design. In general, specific text preprocessing is required before automated topic extraction. Sentences of lesson-learned documents are characterized by the absence of delimiters to mark word boundaries. This study employs the CKIP technology [14] to perform sentence segmentation and text tagging. This technology automatically locates various keywords in sentences of lesson-learned documents and tags them with appropriate morphological feature tags. Before topic extraction, stop words, such as "and" and "the", are deleted from original sentences of a lesson-learned document. Next, a stemming process is conducted with those sentences that don't have stop words. The stemming process cuts the original word down to the root. For example, after the stemming process, the words "design", "designing", and "designs" become the same word, which is "design", because all the words have the same meaning. To stem words, Porter's algorithm was used in the knowledge map system [15]. Once these pre-processes are finished, the module selects among keywords important topic terms and filters out superfluous keywords so that key topic candidate terms can be identified and sent to the next module.

(2) Topic extraction module: Engineers can add or modify an initial topic vocabulary [16], producing a list of important topics that are then defined as the topic vocabulary or thesaurus of lesson-learned documents. The module uses one of the training datasets to construct a topic extraction model, which is then employed to extract topic accurately from test lesson-learned documents.

In this phase, topics are extracted from a set of lessonlearned documents, which can be manually chosen from the documents repository. Each topic will receive a weight using a term weighting algorithm, which is based on the Term Frequency/Inverse Document Frequency (TF/IDF) method [17]. The formula (1) below is the weighting method using TF/IDF.

$$
W_{m t}=\frac{t f_{m t} \log \left(N / n_{t}\right)}{\sqrt{\sum_{t=1}^{k}\left(t f_{m t}\right)^{2}\left[\log \left(N / n_{t}\right)\right]^{2}}}
$$


$W_{m t}$ : weight of topic $t$ in lesson-learned document $m$

$t f_{m t}$ : topic frequency of topic $t$ in document $m$

$N$ : total number of lesson-learned documents

$n_{t}:$ number of documents that contains topic $t$

$k$ : number of topics

After the weighting process, each topic has its own weight. A similar topic can have different weights in different lesson-learned documents due to its frequency in a specific document, since the topic is weighted for each document using the TF/IDF algorithm. If the same topic appears in multiple documents, its weight is determined by the maximum value for all of its weight, according to the formula (2) as follows:

$$
\begin{aligned}
& W_{M t}=\operatorname{Max}\left(W_{D_{m} t}\right) \\
& W_{M t}: \text { maximum value for weight of topic } t \text { in } \\
& \quad \begin{array}{l}
\text { multiple documents } \\
D_{m}: \text { the m-th lesson-learned document } \\
W_{D_{m} t}: \text { weight of topic } t \text { in } D_{m}
\end{array}
\end{aligned}
$$

Once topics are ordered by weights, topics are selected from top-ranked topic list according to the limit that the user set.

(3) Topic relation computation module: Once the toipcs are decided, nodes of the topic knowledge map are gotten, and the main issue lies in how to determine the semantic relations among topics. Semantic relation is denoted by $\operatorname{TS} D\left(t_{i}, t_{j}\right)$. The STKMS utilizes a semantic metric approach that sets two topics with a degree of $[0,1]$. This method is based on the semantic relations among product design domain ontology, so it will be much better than pure topic frequency analysis.

Definition 1 (Semantic relation coefficient (SRC)).

In the product design domain ontology [18]-[20], there are semantic relations between topics. Different semantic relations reflect different semantic distance between topics. They are measured by "Semantic relation coefficient" (SRC), which is between 0 and 1. Table 1 lists some example about semantic relations between topics and their SRCs.

The values assigned for SRCs could be adjusted according to product design experts and knowledge engineers. In the Table 1, SRC of " $i s-a$ " is higher than " $i s-a$ " ", because child nodes inherit all attributes of parent node, while parent node may not involve all attributes of child nodes. So when searching for topics of parent nodes, topics about child nodes could also be shown to users.

Based on above definition, an example of ontology about domain "outfitting design" is illustrated in Fig. 2. The semantic relations and SRCs are also involved in the ontology. In our research, arrows from the source topic to the destination topic represent semantic relevancies, such as "sameas", "similar-with", "is-a", "is-a $a^{-1}$ ", "part-of”, "part-of ${ }^{-1}$ ", "action-object", etc, which are shown in Table 1.

Definition 2 (Topic semantic path (TSP)).

Suppose $t_{1}, \ldots, t_{n}$ are topics, $r_{1}, \ldots, r_{m}$ are semantic relations; From $t_{1}$ to $t_{n}$, there is a semantic path, that is $\exists S R=$
Table 1 Semantic relation coefficient (SRC).

\begin{tabular}{c|c|c}
\hline Semantic relevance & SRC & Example \\
\hline Same-as & 1 & Trestle-same-as $\rightarrow$ Rigger \\
Similar-with & 0.95 & Pipe-similar-with $\rightarrow$ Hose \\
$I s-a$ & 0.9 & Heater pipe- $i s-a \rightarrow$ Pipe \\
$I s-a^{-1}$ & 0.4 & Pipe- is $a^{-1} \rightarrow$ Heater pipe \\
Part-of & 0.7 & Rigger-part-of $\rightarrow$ Pipeline system \\
Part-of ${ }^{1}$ & 0.3 & Pipeline system-part-of ${ }^{1} \rightarrow$ Rigger \\
$\ldots$ & $\ldots$ & $\ldots$ \\
Action-object & 0.2 & Pipefitter-action-object $\rightarrow$ Pipe clamp \\
$\ldots$ & $\ldots$ & $\ldots$ \\
\hline
\end{tabular}

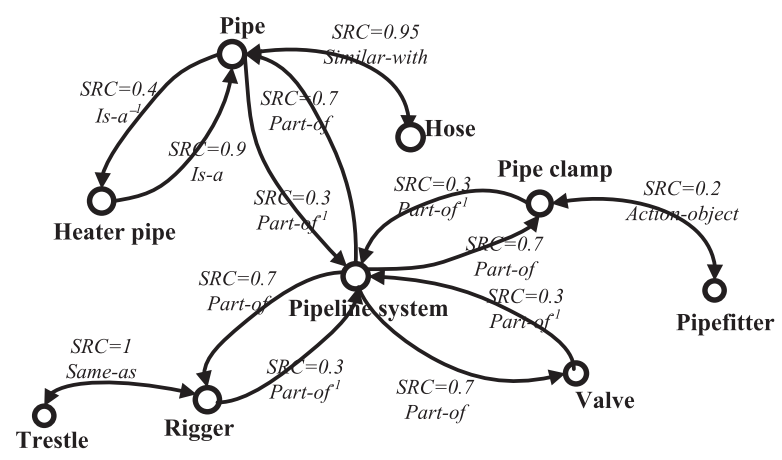

Fig. 2 Part of ontology about "pipeline system of outfitting design".

$\left\{r_{1}, \ldots, r_{m}\right\}$, the set of SRCs is $\rho S=\left\{\rho_{1}, \ldots, \rho_{m}\right\}$, between $t_{1}$ and $t_{n}: \overline{t_{1} r_{1} t_{2} r_{2} \ldots t_{n} r_{m}}$, which is named with "Topic Semantic Path", denoted by $T S P\left(t_{1}, t_{n}\right)$. The product of the SRCs of all semantic relations on the path is defined as "similarity latent value of path", denoted by $F \operatorname{Num}\left(t_{1}, t_{n}\right)=\prod_{i=1}^{m} \rho_{i}$.

Definition 3 (Topic Semantic Distance (TSD)).

Suppose $t_{i}$ and $t_{j}$ are two topics, the "Topic Semantic Distance" is defined as the maximum of semantic distance's values of all the $n$ paths between them. That is shown in formula (3):

$$
\operatorname{TSD}\left(t_{i}, t_{j}\right)=\max _{k=1, \ldots, m}\left[F N u m_{k}\left(t_{i}, t_{j}\right)\right]
$$

Here, $m$ is the number of semantic paths between $t_{i}$ and $t_{j}$. If there is no path between two topics, their TSD is 0 . As to the example of topics in Fig. 2, we could calculate their semantic distance, for example:

$$
\operatorname{TS} D \text { ('Pipefiter', 'Rigger') }=0.2 \times 0.3 \times 0.7=0.042
$$

(4) Topic weight computation module: This module computes the weight of each topic in the topic repository to represent their relative importance in the lesson-learned documents. Topics are described by a set of tuples including topics and its weight: $\left\{\left\langle t_{i}, w_{t_{i}}\right\rangle t_{i} \in T, w_{t_{i}} \in W\right\} ; T$ is a set of pre-designed domain topics, and $W$ is the set of weights for rating the topics. An algorithm called Relation Strength Inverse Sentence Frequency (RSISF) is proposed for the topic weight conversion. In short, it converses the summation of the relation strengths a topic has to other topics into the weight of the topic. RSISF is realized to caculate $w_{t_{i}}$ in the formula (4) as follows. 


$$
\begin{aligned}
& w_{t_{i}}=\sum_{i=1}^{T_{\text {num }}} \sum_{j=1}^{T_{\text {num }}}\left\{\max _{k=1, \ldots, m}\left[F \operatorname{Num}_{k}\left(t_{i}, t_{j}\right)\right]\right\} \times\left(\log \frac{N}{n_{i}}\right) \\
& T_{\text {num }}: \text { Total number of topics in } T \\
& t_{i}: \text { the ith topic in } T \\
& t_{j}: \text { the jth topic in } T, j \neq i \\
& m: \text { total number of semantic paths between } t_{i} \text { and } t_{j} \\
& k: \text { the kth semantic path between } t_{i} \text { and } t_{j} \\
& N: \text { total number of sentences in lesson-learned } \\
& \quad \text { documents } \\
& n_{i}: \text { total number of sentences in which the topic } \\
& \quad \text { i appears together with others in the lesson- } \\
& \quad \text { learned documents. }
\end{aligned}
$$

The higher the topic weight is, the more important the topic is in the lesson-learned documents. The results of the computations are stored in a topic weight database.

(5) Knowledge map generation module: This module generates graphical knowledge maps that display relations and topic nodes using Graphviz technology [21]. Topics identified by the domain topic extraction module are used to generate an annotated semantic-based topic knowledge map, with topic weights and semantic strengths both coming from the topic weight database and the topic relation database. The map concretizes and visualizes the importance of these topics and their relations' strengths. This map depicts the overall structure of all the relevant topics to assist engineers in developing a comprehensive understanding of the topics' distribution among lesson-learned documents and in making decisions about lesson-learned documents selections and learning.

\section{STKMS Implementation}

According to those considerations, we designed the operational semantic-based topic knowledge map system, STKMS. Figure 3 (a) shows a file folder that contains lesson-learned documents in the local network of a shipbuilding company, and the types of documents are Microsoft Word documents. Figure 3 (b) shows content of a lessonlearned document on outfitting design which is written by the design department, each document has main attributes such as: Problem Description, Method Description, Author ID, Author Name, etc. By finding and reading the related lesson-learned documents, engineers can learn from others' methods when facing with similar problems and difficulties.

Figure 4 shows the lesson-learned documents imported interface. In this interface, engineers could upload or add exsiting lesson-learned documents as the input of STKMS. Engineers can click the "Upload Lesson-learned Document" button, and then select multiple files or file folders to import all the corresponding contents of documents at one time. Also, Engineers can click the "Add" button to add new lesson-learned documents one by one, as long as they fill in the relevant information.

The STKMS then conduct an analysis on the lesson-

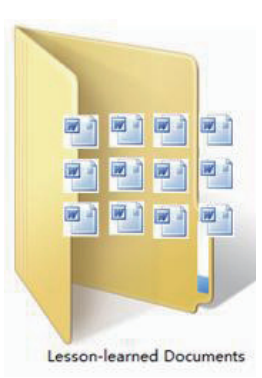

(a) A file folder

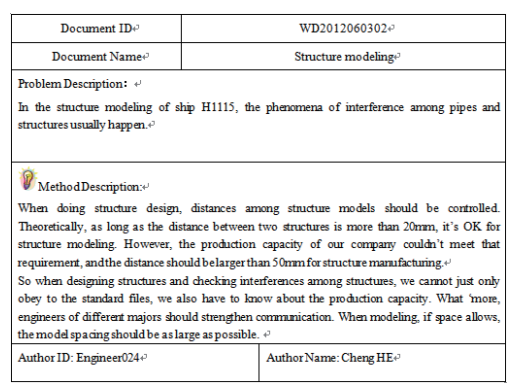

(b) Content of a document
Fig. 3 A file folder and content of a lesson-learned document.

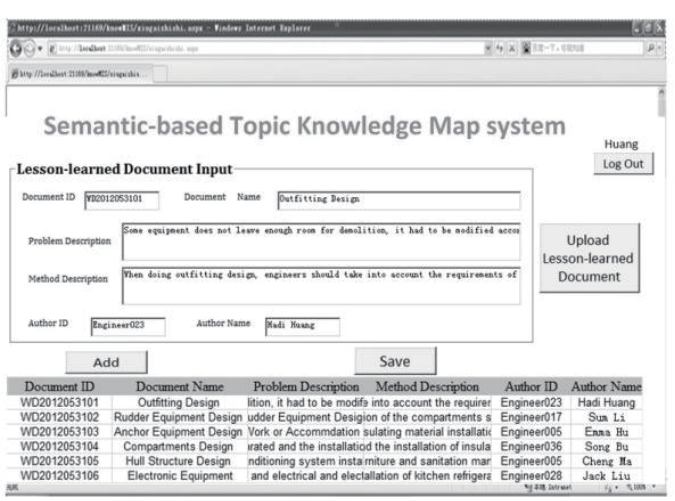

Fig. 4 Lesson-learned documents imported interface.

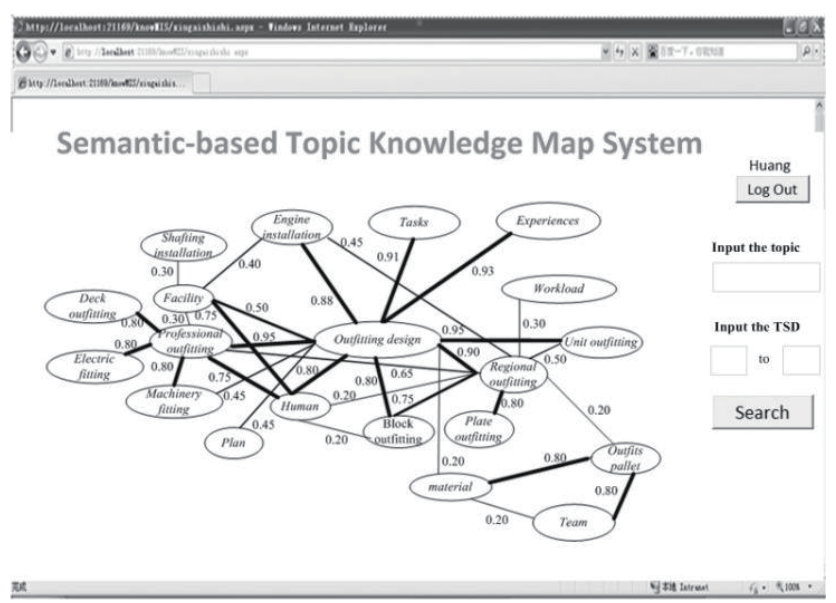

Fig.5 Topic navigation for lesson-learned documents reuse in outfitting design.

learned documents by the knowledge map creation method, such as CKIP processing, morphological features checking, and automatic topic extraction, etc. Then a topic knowledge map is displayed afterwards showing the result.

Figure 5 illustrates the topic knowledge map for the inputed lesson-learned documents. STKMS offers multiple benefits. By exploring the STKMS, an engineer can learn about the topics in outfitting design domain at some level of knowledge without accessing original lesson-learned documents. Furthermore, STKMS can help engineers see a 


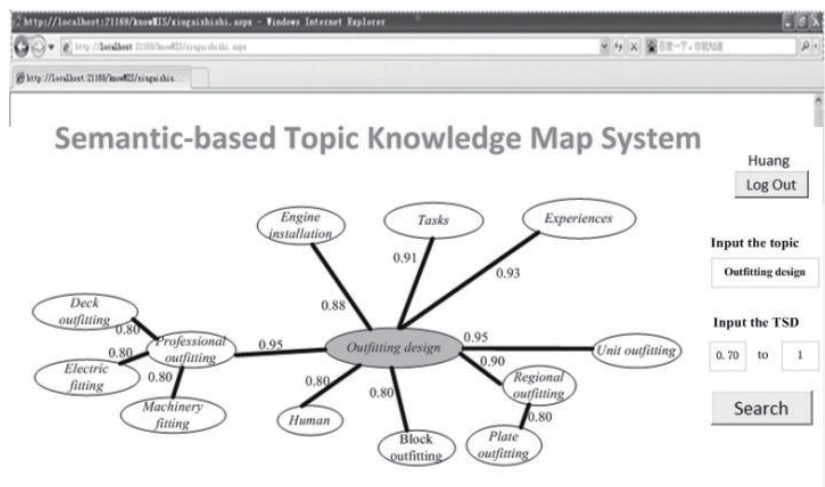

grasiver $6 \cdot \tan$.

Fig. 6 Semantic-based topic search for lesson-learned documents.

holistic picture of all the topics and their relations. The STKMS reveals relations among topics, which allow engineers to identify which topics are related to others, and this is hardly possible when doing browse-based documents finding. When performing an outfitting design work, under the navigation of STKMS an engineer can directly access relevant lesson-learned documents from a certain topic through the hyperlinks. A topic on the map may hyperlink several documents and a lesson-learned document may also connect with several topics.

The nodes in the map are topics extracted by our approach. The semantic relation coefficient (SRC) is showed on the map. Semantic-based topic search for lesson-learned documents is illustrated by Fig. 6. For example, if we input a topic "outfitting design", and set the value of topic semantic distance (TSD) between 0.7 and 1, the topic knowledge map will change and only the nodes that satisfy the conditions will remain.

\section{Evaluation the Quality of Knowledge Map}

\subsection{Evaluation Criteria}

In order to determine the quality of the knowledge map generated by our approach, we conducted an experiment that compared the system with domain experts in assigning lesson-learned documents to specific topics [22].

In this study, precision and recall measures are used. Precision is a measure of the percentage of lesson-learned documents assigned to a topic by our approach and also by a domain expert. Recall is a measure of the percentage of lesson-learned documents assigned to a topic by a domain expert and also by our approach.

For each topic, the precision and recall are defined as that shown in formula (5) and (6).

$$
\begin{aligned}
& \text { precision }=\frac{N_{A \cap M}}{N_{A}} \\
& \text { recall }=\frac{N_{A \cap M}}{N_{M}}
\end{aligned}
$$

Table 2 Precision/recall of the experts.

\begin{tabular}{lccccccccc}
\hline \multicolumn{1}{c}{ Expert Expert Expert Expert Expert Expert Expert Expert Expert } \\
1 & 2 & 3 & 4 & 5 & 6 & 7 & 8 & 9 \\
\hline Precision & 0.86 & 0.91 & 0.91 & 0.97 & 0.96 & 0.98 & 0.91 & 0.92 & 0.93 \\
\hline Recall & 0.88 & 0.88 & 0.93 & 0.96 & 0.97 & 0.98 & 0.92 & 0.91 & 0.93 \\
\hline
\end{tabular}

Where $M$ and $A$ denote the set of lesson-learned documents allocated to a specific topic respectively by a domain expert and our approach. $N_{A}, N_{M}$ and $N_{A \cap M}$ denote respectively the number of lesson-learned documents in $A, M$ and both in $A$ and $M$. The whole precision and recall are calculated by averaging the values of "precision" and "recall" respectively from all topics on the knowledge map.

\subsection{Experimental Design}

We use a lesson-learned document set on outfitting design obtained from a ship-building company. The document set contains 154 lesson-learned documents which are written by engineers from their design experiences. The domain experts are senior engineers on outfitting design. Each expert spends 120 min to finish assigning lesson-learned documents to specific topics on knowledge map generated by our approach, and the cooperation between experts is not allowed. The differences of the system and domain experts in assigning lesson-learned documents to specific topics are analyzed to evaluate the quality of the knowledge map.

\subsection{Evaluation Results}

The "precision" and "recall" are shown in Table 2. We found that the precision and recall are very high (86-98\%); however, the results of lesson-learned documents allocated to specific topics vary greatly among different experts.

It is insightful to modify precision and recall measures to reflect the consensus of results of the allocations by different experts. The revised precision and recall for each topic are defined as that shown in formula (7) and (8):

$$
\begin{aligned}
& \text { revised precision }=\frac{N_{U_{1} \cap U_{2} \ldots U_{i} \ldots \cap U_{n}}}{N_{A}} \\
& \text { revised recall }=\frac{N_{U_{1} \cap U_{2} \ldots U_{i} \ldots \cap U_{n}}}{N_{U_{1} \cup U_{2} \ldots U_{i} \ldots \cup U_{n}}}
\end{aligned}
$$

where $A$ and $U_{i}$ denote the set of lesson-learned documents assigned to a topic respectively by our approach and by both our approach and domain expert $i ; N_{A}$ denotes the number of lesson-learned documents in A, $N_{U_{1} \cup U_{2} \ldots U_{i} \ldots \cup U_{n}}$ denotes the number of distinct lesson-learned documents from $U_{1} \cup U_{2} \ldots U_{i} \ldots \cup U_{n}$, and $N_{U_{1} \cap U_{2} \ldots U_{i} \ldots \cap U_{n}}$ denotes the number of common lesson-learned documents from $U_{1} \cap U_{2} \ldots U_{i} \ldots \cap U_{n}$.

The revised precision is a measure of the percentage of the lesson-learned documents on a specific topic assigned by our approach which are also jointly designated by $n$ domain experts. The revised recall is a measure of the percentage of the distinct lesson-learned documents on a specific topic designated $n$ domain experts which are also jointly assigned to that topic by our approach. The whole knowledge map's 
revised precision and recall are calculated by averaging the revised precision and recall from all topics.

From experiments, we obtain revised precision 0.79 and revised recall 0.76 for knowledge maps in the lessonlearned document set. From these evaluation results of revised precision and recall, the common consensus evaluation of the lesson-learned document sets is lower than those using original precision and recall measures. It implies that our approach enacts a high degree of freedom and presents difficulties in reaching consensus in topic allocation by individual domain experts.

One-way analysis of variance (ANOVA) test was conducted to test if knowledge maps of different subject of lesson-learned documents resulted in significant differences measured in precision and recall. The result shows that no significant difference exists between these knowledge maps. In summary, our approach can achieve effective performance.

\section{Evaluation the Performance of Utilizing STKMS}

\subsection{Experimental Design Method}

In this research, we have to validate the performance of utilizing STKMS. Our hypotheses are as follows:

$\boldsymbol{H}$ : STKMS-based lesson-learned documents learning is more effective than browse-based one on learning performance.

Ha: STKMS-based lesson-learned documents learning is more effective than browse-based one on learning score.

$\boldsymbol{H b}$ : STKMS-based lesson-learned documents learning is more effective than browse-based one on learning satisfaction.

We used a laboratory experimental design to test the hypothesis about learning performance of lesson-learned documents with the STKMS navigation. The participants, measurements, lesson-learned documents, and experiment procedure for the experiment are described in the following sections.

\subsubsection{Participants}

Participants of the experiment are assistant engineers in the field of outfitting design. We have invited 76 assistant engineers who have similar educational backgrounds and working environments to participate in the experiment. They are randomly divided into two groups. There are 38 members in the experimental group (STKMS-based) and 38 members in the control group (browse-based). To increase the reliability of the experiment, the two groups learn with a same set of lesson-learned documents. The experimental group learns with the assistant of STKMS, and the control group learns by browsing lesson-learned documents in a folder stored in hard disk.

\subsubsection{Measurements}

In this experiment, learning performance is measured by learning score and learning satisfaction. The evaluations of learning include comprehensive test sheets in the field of outfitting design. There are several questions for testing the learning of major conception and principles of outfitting design. To increase the content validity of these questions, they are across all lesson-learned documents and further reviewed and modified by experts on outfitting design. One question, for example, asks the following: which of the following does not belong to the category of regional outfitting? (A) Unit outfitting (B) Block outfitting (C) Plate outfitting (D) Machinery fitting.

The post-test questionnaire measures learning satisfaction. For the measure of learning satisfaction, we use Likert's 7-point scale as raised by Sun and Cheng [23].

\subsubsection{Lesson-Learned Documents}

The lesson-learned documents used in the control group are derived from the design department of a famous shipbuilding company in China, and they are just stored in a folder of the local network. The lesson-learned documents for the experimental group are the same sources as control group, but represented with STKMS (like Fig. 4).

\subsubsection{Experiment Procedure}

The experiment is done simultaneously in the experimental and control groups and takes about one and half hours to complete. Participants spend about $10 \mathrm{~min}$ for explanation of the operations, $50 \mathrm{~min}$ for self-learning and $30 \mathrm{~min}$ for the post-test. The allocation for the experiment schedule is listed in Table 3. The design of experiment procedure, measurements, and questionnaires in this study both refers to previous studies and reviewed by experts in related fields to increase the validity of experiment design [24]. The statistical methods applied include KR-20 [25] and Cronbach's

Table 3 Allocation of experiment schedule.

\begin{tabular}{|c|c|c|}
\hline Phases & Experimental group & Control group \\
\hline Preparation & $\begin{array}{l}\text { - Explanation of "STKMS- } \\
\text { based user guide" } \\
\text { - Time: 10min }\end{array}$ & $\begin{array}{l}\text { - Explanation of "browse- } \\
\text { based user guide" } \\
\text { - Time: } 10 \mathrm{~min}\end{array}$ \\
\hline Learning & $\begin{array}{l}\text { - Method: self-learning } \\
\text { - Material: learns with the } \\
\text { assistant of STKMS } \\
\text { - Time: } 50 \text { min }\end{array}$ & $\begin{array}{l}\text { - Method: self-learning } \\
\text { - Material: browsing lesson- } \\
\text { learned documents stored in } \\
\text { a folder of local network } \\
\text { - Time: } 50 \mathrm{~min}\end{array}$ \\
\hline Post-test & $\begin{array}{l}\text { - Testing after learning and } \\
\text { fill out a post-test } \\
\text { questionnaire } \\
\text { - Time: } 30 \mathrm{~min}\end{array}$ & $\begin{array}{l}\text { - Testing after learning and } \\
\text { fill out a post-test } \\
\text { questionnaire } \\
\text { - Time: } 30 \mathrm{~min}\end{array}$ \\
\hline
\end{tabular}


Table 4 Item difficulty and discrimination for the questions.

\begin{tabular}{l|l|l}
\hline Number & Difficulty (.2-.8) & Item discrimination $(>.3)$ \\
\hline S-1 & 0.47 & 0.52 \\
S-2 & 0.66 & 0.53 \\
S-3 & 0.56 & 0.47 \\
S-4 & 0.14 & 0.15 \\
S-5 & 0.60 & 0.51 \\
S-6 & 0.71 & 0.56 \\
S-7 & 0.43 & 0.50 \\
S-8 & 0.52 & 0.53 \\
S-9 & 0.58 & 0.47 \\
S-10 & 0.38 & 0.42 \\
S-11 & 0.46 & 0.42 \\
S-12 & 0.58 & 0.55 \\
S-13 & 0.13 & 0.14 \\
F-1 & 0.06 & 0.06 \\
F-2 & 0.11 & 0.13 \\
F-3 & 0.25 & 0.38 \\
F-4 & 0.36 & 0.40 \\
F-5 & 0.32 & 0.37 \\
S: multiple-choice question & Fill-in-blank question
\end{tabular}

$\alpha$ to test the internal consistency of the comprehensive test sheet and the post-test questionnaire respectively, one-way ANOVA and regression analysis. SPSS tools are used for analysis.

\subsection{Evaluation Results and Data Analysis}

\subsubsection{Reliability of Comprehensive Test Sheet}

Since scores of questions in comprehensive test sheet are used as the basis for learning evaluation, the reliability of the questions is validated by the measure of Kuder-Richardson Formula 20 (KR-20) [25]. In the original comprehensive test sheet, there are 13 multiple-choice and 5 fill-in-blank questions. Through KR-20 methods [25], results of item difficulty and discrimination for each question are calculated, which are shown in the Table 4. According to the suggestion of Xue [26], the difficulty of questions between the level 0.2 and 0.8 will be better, and the discrimination of questions is greater than 0.3 will be better. The discrimination and difficulty of questions S-4, S-13, F-1 and F-2 in Table 4 are not matched with the requirement above. After the deletion of these four questions, the KR-20 for the 14 questions is 0.78 , greater than the water mark of 0.70 suggested by Nunnally [27].

\subsubsection{Reliability of Post-Test Questionnaire}

The reliability coefficient of Cronbach's $\alpha$ for constructs of learning satisfaction in the post-test questionnaire is 0.91, which exceeds the standard threshold of 0.70 .

\subsubsection{Hypothesis Testing of Learning Performance}

Hypothesis H suggests that STKMS-based lesson-learned documents learning is more effective than browse-based one on learning performance. Learning performance is further subdivided as learning score ( $\mathrm{Ha}$ ) and learning satisfaction $(\mathrm{Hb})$.
Table 5 Descriptive statistics for the learning score of two groups.

\begin{tabular}{lcccccc}
\hline & \multirow{2}{*}{ N Mean } & $\begin{array}{c}\text { Std. } \\
\text { deviation }\end{array}$ & $\begin{array}{c}\text { Std. } \\
\text { error }\end{array}$ & & $\begin{array}{c}\mathbf{9 5 \%} \text { Confidence interval } \\
\text { for mean }\end{array}$ \\
\cline { 5 - 7 } & & & & Lower bound & Upper bound \\
\hline Browse-based & 38 & 11.53 & 2.78 & 0.323 & 10.65 & 12.41 \\
STKMS-based & 38 & 14.80 & 3.56 & 0.367 & 13.67 & 15.93 \\
Total & 76 & 13.11 & 3.23 & 0.296 & 12.38 & 13.84 \\
\hline
\end{tabular}

Table 6 ANOVA analysis for learning score.

\begin{tabular}{lccccc}
\hline Dependent variable: learning score & & & \\
\hline & $\begin{array}{l}\text { Sum of } \\
\text { squares }\end{array}$ & df & $\begin{array}{c}\text { Mean } \\
\text { square }\end{array}$ & F-value & Significance \\
\hline Between groups & 130.234 & 1 & 130.234 & 15.358 & 0.000 \\
Within groups & 627.532 & 74 & 8.480 & & \\
Total & 757.766 & 75 & & & \\
\hline
\end{tabular}

Table 7 Descriptive statistics for the learning satisfaction of two groups.

\begin{tabular}{|c|c|c|c|c|c|c|}
\hline & \multirow[t]{2}{*}{$\mathbf{N}$} & \multirow[t]{2}{*}{ Mean } & \multirow[t]{2}{*}{$\begin{array}{c}\text { Std. } \\
\text { deviation }\end{array}$} & \multirow[t]{2}{*}{$\begin{array}{l}\text { Std. } \\
\text { error }\end{array}$} & \multicolumn{2}{|c|}{$\begin{array}{l}95 \% \text { Confidence interval } \\
\text { for mean }\end{array}$} \\
\hline & & & & & Lower bound & Upper bound \\
\hline Browse-based & 38 & 17.56 & 4.235 & 0.354 & 16.21 & 18.91 \\
\hline STKMS -based & 38 & 22.54 & 4.856 & 0.376 & 21.01 & 24.08 \\
\hline Total & 76 & 20.38 & 5.326 & 0.382 & 19.18 & 21.58 \\
\hline
\end{tabular}

Table 8 ANOVA analysis for learning satisfaction.

\begin{tabular}{lccccc}
\hline \multicolumn{6}{l}{ Dependent variable: learning satisfaction } \\
\hline & $\begin{array}{c}\text { Sum of } \\
\text { squares }\end{array}$ & df & $\begin{array}{c}\text { Mean } \\
\text { square }\end{array}$ & F-value & Significance \\
\hline Between groups & 362.581 & 1 & 362.581 & 11.108 & 0.000 \\
Within groups & 2415.520 & 74 & 32.642 & & \\
Total & 2778.101 & 75 & & & \\
\hline
\end{tabular}

This research applies a one-way ANOVA to test Ha and $\mathrm{Hb}$. The results of the one-way ANOVA for Ha are shown in Tables 5 and 6, and the one-way ANOVA for $\mathrm{Hb}$ are shown in Tables 7 and 8. In Table 5, the average learning score of STKMS-based lesson-learned documents learning (experimental group) and browse-based lesson-learned documents learning (control group) are 14.80 and 11.53. Table 6 shows that these differences are significant $(\mathrm{F}(1,74)=15.358, \mathrm{P}<$ 0.001). Thus, findings support hypothesis Ha. The STKMSbased lesson-learned documents learning could help engineers attain higher scores than the browse-based one.

In Table 7, the average learning satisfaction of STKMS-based lesson-learned documents learning and browse-based lesson-learned documents learning are 22.54 and 17.56. In Table 8, results show this difference is significant $(\mathrm{F}(1,74)=11.108, \mathrm{P}<0.001)$. Hypothesis $\mathrm{Hb}$ is sta- 
tistically supported. The STKMS-based lesson-learned documents learning could help learners acquire higher learning satisfaction than browse-based ones.

Findings from Tables 5-8 suggest that the learning performance of STKMS-based lesson-learned documents learning is more effective than browse-based one.

\subsubsection{Discussion}

In this research, we construct STKMS, and utilize it for lesson-learned documents reusing and test its utilizing performance. There are some studies that emphasize developing tools for constructing graphic-based materials, such as CmapTools developed by [28]. These studies however have not tested the learning performance of materials with those developed tools. This research constructs STKMS for lesson-learned documents reuse and finds that it can improve the performance of learning. These results of this research could make breakthrough and give a big change from existing browse-based lesson-learned documents learning style to STKMS based one to gain the better learning performance.

There are also some limitations. For example, Table 5 shows that the standard deviation of the experimental group is higher than that of the control group $(3.56,2.78)$, which means the scores of the experimental group is more dispersed than scores of the control group. This suggests that some participants may not comprehend the meaning and usage of STKMS well. Training users to apply the technology and design more user-friendly interface of STKMS are significant issues for the future.

\section{Conclusion}

In conclusion, this study presents a novel approach method to build a STKMS for lesson-learned documents reuse in product design. The results of experiments show that our method of knowledge map creation is effective and the utilization of STKMS in lesson-learned documents learning and reuse has positive effects on performance.

However, there are some limitations for the current model and methods, which need to be studied in the future:

$\diamond$ The main limitation is that engineers have to spend more time understand the definitions of components and their relationships between components in STKMS. Training users to apply the technology and design more user-friendly interface of STKMS are significant issues for the future.

$\diamond$ How to make the STKMS involves much more semantic reasoning capacities. It needs us to study the deep semantic relations among the topics in outfitting design domain.

\section{Acknowledgments}

The research is supported by National Nature Science Foundation of China (No. 70971085, 71271133), the Research
Fund for the Doctoral Program of Higher Education of China (No. 20100073110035), and Innovation Program of Shanghai Municipal Education Commission (13ZZ012). The authors would like to thank all the participants for their efforts in our experiments and the viewers for their helpful comments.

\section{References}

[1] A. Lee, H. Chen, and Y. Tong, "Developing new products in a network with efficiency and innovation," Int. J. Production Research, vol.46, no.17, pp.4687-4707, 2008.

[2] A. Gunasekaran and E. Ngai, "Knowledge management in 21st century manufacturing," Int. J. Production Research, vol.45, no.11, pp.2391-2418, 2007.

[3] B. Song and Z. Jiang, "Proactive search enabled context-sensitive knowledge supply situated in computer-aided engineering," Advanced Engineering Informatics, 212.

[4] K. Steiner, W. Essmayr, and R. Wagner, "Topic maps-an enabling technology for knowledge management," Proc. 12th International Workshop on Database and Expert Systems, Applications, 2001.

[5] B. Le Grand and M. Soto, "Visualisation of the semantic web: topic maps visualisation in Information Visualisation," Proc. IEEE Sixth International Conference on 2002.

[6] K. Böhm, et al., Topic map generation using text mining, J. UCS, vol.8, no.6, pp.623-643, 2002.

[7] L. Stanescu and D. Burdescu, "Information structuring and retrieval with topic maps for medical E-learning," Advances in Electrical and Computer Engineering, vol.9, no.3, pp.27-33, 2009.

[8] L. Stanescu, et al., "A software system for viewing and querying automatically generated Topic Maps in the e-learning domain," Informatica (Ljubljana), vol.34, no.4, pp.441-450, 2010.

[9] G. Mihai, et al., "A topic map for "subject-centric"," learning, G.A. Papadopoulos, Editor, pp.141-150, 2009.

[10] M. Rafi and M.S. Shaikh, "An improved semantic similarity measure for document clustering based on topic maps," arXiv preprint arXiv:1303.4087, 2013.

[11] N. Ellouze, N. Lammari, and E. Méais, "CITOM: An incremental construction of multilingual topic maps," Data and Knowledge Engineering, vol.74, pp.46-62, 2012.

[12] H. Lu and G. Liu, "Text visualization and visual analytics based on multi-layer topic maps,' J. Information and Computational Science, vol.8, no.12, pp.2459-2464, 2011.

[13] D. Newman, et al., "Visualizing search results and document collections using topic maps," Journal Web Semantics, vol.8, no.23, pp.169-175, 2010.

[14] W.-Y. Ma and K.-J. Chen, "Introduction to CKIP Chinese word segmentation system for the first international Chinese Word Segmentation Bakeoff," Proc. Second SIGHAN Workshop on Chinese Language Processing-Vol.17, 2003 Association for Computational Linguistics.

[15] M.F. Porter, "An algorithm for suffix stripping," Program: Electronic Library and Information Systems, vol.14, no.3, pp.130-137, 1980.

[16] Y. Huang, et al., "Design of ship-block production scheduling oriented knowledge navigation system," J. Chongqing University, vol.1, p.8, 2013.

[17] G. Salton and C. Buckley, "Term-weighting approaches in automatic text retrieval," Information Processing \& Management, vol.24, no.5, pp.513-523, 1988.

[18] L. Zhen, G.Q. Huang, and Z. Jiang, "Recommender system based on workflow-Decision Support Systems," Decision Support Systems, vol.48, no.1, pp.237-245, 2009.

[19] L. Zhen, L. Wang, and J.-G. Li, "A design of knowledge management tool for supporting product development," Information Processing \& Management, vol.49, no.4, pp.884-894, 2013.

[20] L. Zhen, Z. Jiang, and H. Song, "Distributed recommender for peer- 
to-peer knowledge sharing," Information Sciences, vol.18, no.8, pp.3546-3561, 2010.

[21] E.R. Gansner and S.C. North, "An open graph visualization system and its applications to software engineering," Software Practice and Experience, vol.3, no.11, pp.1203-1233, 2000.

[22] F. Lin and C. Hsueh, "Knowledge map creation and maintenance for virtual communities of practice," Information Processing \& Management, vol.42, no.2, pp.551-568, 2006.

[23] P.-C. Sun and H.K. Cheng, "The design of instructional multimedia in e-Learning: A Media Richness Theory-based approach," Computers \& Education, vol.49, no.3, pp.662-676, 2007.

[24] S. Ruey-Shiang, "A study of learning performance of e-learning materials design with knowledge maps," Computers \&amp; Education, vol.54, no.1, pp.253-264, 2010.

[25] G.F. Kuder and M.W. Richardson, "The theory of the estimation of test reliability," Psychometrika, vol.2, no.3, pp.151-160, 1937.

[26] W. Xue, SPSS statistical analysis and application, Electronic Industry Press, Beijing, 2009.

[27] J.C. Nunnally, Psychometric Theory 3E, Tata McGraw-Hill Education, 2010.

[28] A.J. Cañas, et al., "CmapTools: A knowledge modeling and sharing environment in Concept maps: Theory, methodology technology," Proc. the First International Conference on Concept Mapping, 2004.

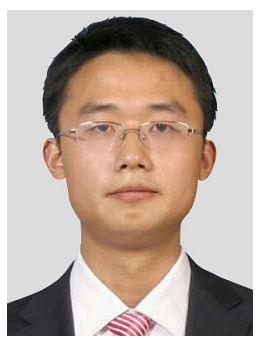

Ywen Huang is a PH.D. candidate at the Department of Industrial Engineering, Shanghai Jiao Tong University, P.R.C. His current research interests include: knowledge management, knowledge demand and knowledge navigation. He has published 8 papers in referred international conferences and journals.

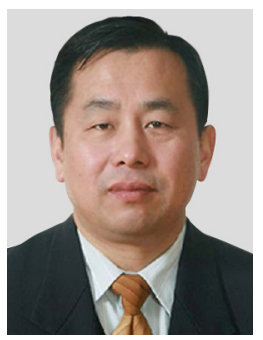

Zhua Jiang is a professor at the Department of Industrial Engineering, Shanghai Jiao Tong University, P.R.C. His current research interests include: knowledge management, and product design. He has published 63 papers in referred International conferences and journals, including $18 \mathrm{SCI}$ journal papers and $25 \mathrm{EI}$ journal papers. 